PROCEEDINGS OF THE

AMERICAN MATHEMATICAL SOCIETY

Volume 135, Number 5, May 2007, Pages 1263-1269

S 0002-9939(06)08637-0

Article electronically published on November 13, 2006

\title{
VARIETIES WITH A REDUCIBLE HYPERPLANE SECTION WHOSE TWO COMPONENTS ARE HYPERSURFACES
}

\author{
JOSÉ CARLOS SIERRA AND ANDREA LUIGI TIRONI
}

(Communicated by Michael Stillman)

\begin{abstract}
We classify smooth complex projective varieties $X \subset \mathbb{P}^{N}$ of dimension $n \geq 2$ admitting a divisor of the form $A+B$ among their hyperplane sections, both $A$ and $B$ of codimension $\leq 1$ in their respective linear spans. In this setting, one of the following holds: 1) $X$ is either the Veronese surface in $\mathbb{P}^{5}$ or its general projection to $\left.\mathbb{P}^{4}, 2\right) n \leq 3$ and $X \subset \mathbb{P}^{n+2}$ is contained in a quadric cone of rank 3 or 4,3$) n=2$ and $X \subset \mathbb{P}^{3}$.
\end{abstract}

\section{INTRODUCTION}

Let $X \subset \mathbb{P}^{N}$ be an irreducible, nondegenerate, smooth projective variety over $\mathbb{C}$ of dimension $n \geq 2$, embedded by a very ample divisor $H$ on $X$.

In 3], Chandler, Howard and Sommese started to study pairs $(X, H)$ as above for $n \geq 4$ when $|H|$ contains an element of type $A+B$, with $A, B$ smooth irreducible divisors meeting transversally and of sectional genus zero. They obtained the following possibilities: 1) $X$ is a rational scroll of $(n-1)$-planes embedded by its tautological line bundle, 2) $X$ is the Segre embedding of $\left.\mathbb{P}^{2} \times \mathbb{P}^{2}, 3\right) X \cong \mathbb{P}(\mathcal{E})$, where $\mathcal{E} \cong \mathcal{O}_{\mathbb{P}^{2}}(1)^{\oplus 2} \oplus \mathcal{O}_{\mathbb{P}^{2}}(2)$ and $H$ is the tautological line bundle or 4$) X$ is a Mukai 4-fold. This result was slightly improved in [5] excluding case 4).

Moreover, for $n=3$, the list of all possible numerical invariants concerning $A, B$ and $A \cap B$, when both $A$ and $B$ are rational scrolls meeting along a smooth curve of positive genus, is provided in [2]. A detailed classification result when $H$ is only an ample and spanned line bundle and $A \cap B$ is also a rational curve is given in [6].

The technics used in the quoted papers are typical of the adjunction theory, but the use of the adjunction mapping seems not to be a powerful tool in low dimensions. On the other hand, one of the advantages of the adjunction theory is that sometimes we can consider $H$ only as an ample and spanned line bundle (see [3] and [6]).

In this paper our approach is different. Let $H$ be a very ample divisor on $X$ and consider the embedding $X \subset \mathbb{P}(V)=\mathbb{P}^{N}$ given by a very ample linear system $V \subseteq H^{0}\left(X, \mathcal{O}_{X}(H)\right)$ (that is, we are not assuming $X \subset \mathbb{P}^{N}$ to be linearly normal). Suppose that $|V|$ contains a divisor of the form $A+B$, with $A, B$ effective. We will

Received by the editors January 21, 2005 and, in revised form, December 6, 2005.

2000 Mathematics Subject Classification. Primary 14C20; Secondary 14N05.

Key words and phrases. Algebraic geometry, reducible hyperplane sections of varieties.

This work was done in the framework of the National Research Project "Geometry on Algebraic Varieties", supported by the MIUR of the Italian Government (Cofin 2002).

(C)2006 American Mathematical Society Reverts to public domain 28 years from publication 
study the case in which both $A$ and $B$ (embedded by $V$ ) have low codimension in their linear spans. Let us note that by this geometric assumption we can work in a more general setting than in [2], [3, [5] and [6], since we consider $n \geq 2$ and we do not impose any restriction on the divisors $A, B$. In particular, we do not require a priori that both components are either smooth or irreducible.

To be precise, we will concentrate on the cases in which one of the divisors, say $A$, is either a linear space (Section 2) or a hypersurface (Section 3). We get the main result of the paper if also $B$ is either a linear space or a hypersurface: 1) $X$ is either the Veronese surface in $\mathbb{P}^{5}$ or its general projection to $\left.\mathbb{P}^{4}, 2\right) n \leq 3$ and $X \subset \mathbb{P}^{n+2}$ is contained in a quadric cone of rank 3 or 4,3$) n=2$ and $X \subset \mathbb{P}^{3}$ (see Corollary 3.5).

The authors would like to thank Professor A. Lanteri for useful comments and suggestions during the preparation of the final version of this paper.

\section{Notation AND BACKGROUnd MATERial}

Let $X \subset \mathbb{P}(V)=\mathbb{P}^{N}$ be an irreducible, nondegenerate, smooth projective variety over $\mathbb{C}$ of dimension $n \geq 2$ (from now on $n$-fold) embedded by a very ample linear system $V \subseteq H^{0}\left(X, \mathcal{O}_{X}(H)\right)$, where $H$ is a very ample divisor on $X$.

Note that the general element of $|V|$ is a smooth divisor by the following wellknown theorem.

Theorem 1.1 (Bertini). The general element of a linear system $|V|$ on $X$ is smooth away from the base locus of $|V|$.

Remark 1.2. A divisor $D \in|V|$ on $X$ is singular at $x \in X$ if and only if the corresponding hyperplane $h_{D} \in \mathbb{P}(V)^{*}$ contains the embedded tangent $n$-plane $\mathbb{T}_{x} X$ to $X$ at $x$.

We suppose that $X$ has a reducible hyperplane section, that is, an element $A+$ $B \in|V|$ with $A, B$ effective divisors. Let us recall the definition of $m$-connectedness for $n=2$.

Definition 1.3. An effective divisor $D$ on a surface is called (numerically) $m$ connected if for every splitting $D=D_{1}+D_{2}$ with $D_{1}, D_{2}$ effective, the inequality $D_{1} \cdot D_{2} \geq m$ holds.

Remark 1.4. A very ample divisor is always 1-connected.

Theorem 1.5 (Van de Ven [8]). Let $X$ be a smooth surface and $H$ a very ample divisor on $X$. If $H$ is not 2-connected, then $X$ is either the Veronese surface or a scroll of lines.

We recall some topological results about the Picard group Pic $X$ of a smooth projective variety $X \subset \mathbb{P}^{N}$.

Theorem 1.6. Let $X \subset \mathbb{P}^{N}$ be an $n$-fold and consider the restriction homomorphism $i_{k}: H^{k}\left(\mathbb{P}^{N}, \mathbb{C}\right) \rightarrow H^{k}(X, \mathbb{C})$. Then $i_{k}$ is an isomorphism in the following situations:

a) (Lefschetz) $X$ is a complete intersection and $k \leq n-1$;

b) (Barth-Larsen [1, [7]) $k \leq 2 n-N$.

The same is valid if we replace $\mathbb{C}$ by $\mathbb{Z}$. 
The above theorem shows two cases in which Pic $X$ is generated by the hyperplane section.

Corollary 1.7. Let $X \subset \mathbb{P}^{N}$ be an $n$-fold. Then $\operatorname{Pic} X \cong \mathbb{Z}$ (generated by the hyperplane section) in the following cases:

a) $X$ is a complete intersection and $n \geq 3$;

b) $n \geq \frac{N+2}{2}$.

Proof. Both a) and b) respectively follow by applying Theorem 1.6 to the exact sequence of sheaves

$$
0 \rightarrow \mathbb{Z} \rightarrow \mathcal{O} \rightarrow \mathcal{O}^{*} \rightarrow 0
$$

having in mind that $\operatorname{Pic} X \cong H^{1}\left(X, \mathcal{O}_{X}^{*}\right)$.

Remark 1.8. In our setting there exists a reducible divisor $A+B \in|V|$, so Pic $X$ is not generated by the hyperplane section. Therefore $n<\frac{N+2}{2}$ and $X \subset \mathbb{P}^{N}$ cannot be a complete intersection (from now on c.i.) if $n \geq 3$.

Denote by $\langle A\rangle \subset \mathbb{P}(V)$ (resp. $\langle B\rangle \subset \mathbb{P}(V)$ ) the linear span of $A$ (resp. $B$ ) embedded by $|V|$. We can suppose

$$
\operatorname{dim}\langle A\rangle \leq \operatorname{dim}\langle B\rangle
$$

throughout. We will study the boundary cases in which $A \subset\langle A\rangle$ has the lowest possible codimension. More precisely we will analyze two possibilities, namely $A=\mathbb{P}^{n-1}$ and $\langle A\rangle=\mathbb{P}^{n}$.

\section{The CASE $A=\mathbb{P}^{n-1}$}

Let $X \subset \mathbb{P}(V)=\mathbb{P}^{N}$ be an $n$-fold having a reducible divisor $A+B \in|V|$. Set $L=\langle A\rangle \cap\langle B\rangle$.

Lemma 2.1. $\operatorname{dim} L \geq n-2$.

Proof. If $\operatorname{dim} L<n-2$, we obtain a very ample divisor on a smooth surface which is not 1-connected by intersecting $X$ with $n-2$ general hyperplanes, contradicting Remark 1.4

We start now with the case $A=\mathbb{P}^{n-1}$. Then either $\operatorname{dim} L=n-2$ or $\operatorname{dim} L=$ $n-1$. Suppose $\operatorname{dim} L=n-2$. Let us prove that this situation characterizes rational scrolls of $(n-1)$-planes.

Example 2.2. Let $0 \leq a_{1} \leq \cdots \leq a_{n}$ be integers and set $\mathcal{E} \cong \bigoplus_{i=1}^{n} \mathcal{O}_{\mathbb{P}^{1}}\left(a_{i}\right)$. A rational scroll of $(n-1)$-planes is the image of $\mathbb{P}(\mathcal{E})$ in $\mathbb{P}(V)$ embedded by a linear system $V \subseteq H^{0}(\mathbb{P}(\mathcal{E}), \mathcal{O}(1))$, where $\mathcal{O}(1):=\mathcal{O}_{\mathbb{P}(\mathcal{E})}(1)$ is the tautological line bundle. Its degree is $d=\sum_{i=1}^{n} a_{i}$ and it is smooth only if $a_{1}>0$. Otherwise, if $0=a_{k}<a_{k+1}$, it is a cone over a smooth rational scroll of $(n-k-1)$-planes with vertex $\mathbb{P}^{k-1}$. We refer to a rational normal scroll of $(n-1)$-planes if it is embedded by the complete linear system $|\mathcal{O}(1)|$. In this case $\mathbb{P}(\mathcal{E}) \subset \mathbb{P}^{d+n-1}$. For a fiber $f=\mathbb{P}^{n-1}$, we have that $\xi=\mathcal{O}(1)-f$ is an effective divisor, so $f+\xi \in|\mathcal{O}(1)|$ and $f \cap\langle\xi\rangle=\mathbb{P}^{n-2}$.

Proposition 2.3. Let $X \subset \mathbb{P}(V)=\mathbb{P}^{N}$ be an $n$-fold having a reducible divisor $A+B \in|V|$. If $A=\mathbb{P}^{n-1}$ and $\operatorname{dim} L=n-2$, then $X$ is a rational scroll of $(n-1)$-planes. 
Proof. If $A=\mathbb{P}^{n-1}$ and $\operatorname{dim} L=n-2$, then it follows from the Grassmann formula $\operatorname{dim}\langle A+B\rangle=\operatorname{dim}\langle A\rangle+\operatorname{dim}\langle B\rangle-\operatorname{dim} L$

that $\operatorname{dim}\langle B\rangle=N-2$, so $|V-B|$ is a pencil of linearly equivalent divisors on $X$. For $\lambda \in \mathbb{P}^{1}$, let $R_{\lambda} \in|V-B|$ and $D \in|V|$. Since $A \in|V-B|$, then

$$
\operatorname{deg} R_{\lambda}=R_{\lambda} \cdot D^{n-1}=A \cdot D^{n-1}=1,
$$

so $R_{\lambda}=\mathbb{P}^{n-1}$ and $X \subset \mathbb{P}^{N}$ is embedded as a rational scroll of $(n-1)$-planes.

Remark 2.4. On the other hand, if $\operatorname{dim} L=n-1$ (equivalently $A \subset\langle B\rangle$ ), we cannot be assured that $A$ moves on $X$. We can only handle this situation if $\operatorname{dim}\langle B\rangle \leq n$.

Corollary 2.5. Let $X \subset \mathbb{P}(V)=\mathbb{P}^{N}$ be an $n$-fold having a reducible divisor $A+B \in$ $|V|$. If $A=\mathbb{P}^{n-1}$ and $\operatorname{dim}\langle B\rangle \leq n$, then one of the following holds:

i) $X \cong \mathbb{P}\left(\mathcal{O}_{\mathbb{P}^{1}}(1) \oplus \mathcal{O}_{\mathbb{P}^{1}}(2)\right) \subset \mathbb{P}^{4}$ or $X \cong \mathbb{P}\left(\mathcal{O}_{\mathbb{P}^{1}}(1)^{\oplus 3}\right) \subset \mathbb{P}^{5}$;

ii) $n=2$ and $X \subset \mathbb{P}^{3}$.

Proof. If $\operatorname{dim} L=n-2$ and $\operatorname{dim}\langle B\rangle=n$, then we get

$$
\operatorname{dim}\langle A+B\rangle=\operatorname{dim}\langle A\rangle+\operatorname{dim}\langle B\rangle-\operatorname{dim} L=n+1
$$

So $X \subset \mathbb{P}^{n+2}$ is a rational scroll of $(n-1)$-planes by Proposition 2.3. Since an $n$-fold $X \subset \mathbb{P}^{n+2}$ other than the projected Veronese surface in $\mathbb{P}^{4}$ is linearly normal by Zak's theorem (see [9], II Cor. 2.17), we deduce that $d=\operatorname{deg} X=3$ (cf. Example 2.2.) and case i) occurs.

Finally, if either $\operatorname{dim} L=n-1$ or $\operatorname{dim}\langle B\rangle=n-1$, then $X \subset \mathbb{P}^{n+1}$ is a hypersurface and $n=2$ follows from Remark 1.8 ,

\section{The CASE $\langle A\rangle=\mathbb{P}^{n}$}

Now suppose $\langle A\rangle=\mathbb{P}^{n}$, that is, $A$ is a hypersurface. Then $n-2 \leq \operatorname{dim} L \leq n$ by Lemma 2.1. If $\operatorname{dim} L<n$, we prove that $A$ moves on $X$ and we give a precise description of $X \subset \mathbb{P}^{N}$.

Example 3.1 (Veronese surface). Embed $\mathbb{P}^{2}$ in $\mathbb{P}^{5}$ by $V=H^{0}\left(\mathbb{P}^{2}, \mathcal{O}_{\mathbb{P}^{2}}(2)\right)$. Then there exists a divisor $A+B \in|V|$ with $A, B \in\left|\mathcal{O}_{\mathbb{P}^{2}}(1)\right|$. Moreover $A \cdot B=1$. The same holds for the projected Veronese surface, that is, $\mathbb{P}^{2}$ embedded in $\mathbb{P}^{4}$ by a general subspace of $H^{0}\left(\mathbb{P}^{2}, \mathcal{O}_{\mathbb{P}^{2}}(2)\right)$ of codimension one.

Example 3.2. Using the notation of Example 2.2, let $\mathbb{P}(\mathcal{E}) \subset \mathbb{P}(V)$ be a rational scroll of $n$-planes embedded by $V \subseteq H^{0}(\mathbb{P}(\mathcal{E}), \mathcal{O}(1))$. Consider a smooth effective divisor $X$ on $\mathbb{P}(\mathcal{E})$ embedded by $\left|\bar{V}_{\mid X}\right|$. Set $A=f_{\mid X}$ and $B \in\left|(V-f)_{\mid X}\right|$. Then $X \subset \mathbb{P}\left(V_{\mid X}\right)$ and $A+B \in\left|V_{\mid X}\right|$. Furthermore $\langle A\rangle \cap\langle B\rangle=\mathbb{P}^{n-1}$ if $\langle A\rangle=f=\mathbb{P}^{n}$.

Proposition 3.3. Let $X \subset \mathbb{P}(V)=\mathbb{P}^{N}$ be an $n$-fold having a reducible divisor $A+B \in|V|$. Suppose $\langle A\rangle=\mathbb{P}^{n}$ and $\operatorname{dim}\langle B\rangle \geq n$. Set $L=\langle A\rangle \cap\langle B\rangle$. Then

a) $X \subset \mathbb{P}^{5}$ is the Veronese surface if $\operatorname{dim} L=n-2$;

b) $X \subset \mathbb{P}^{N}$ is a divisor on a rational scroll of $n$-planes if $\operatorname{dim} L=n-1$.

Proof. Suppose first that $\operatorname{dim} L=n-2$. We start by considering $n=2$. Then $\operatorname{dim} L=0$ and hence $A \cdot B=1$, so $X$ is not 2-connected. Moreover

$$
\operatorname{dim}\langle A+B\rangle=\operatorname{dim}\langle A\rangle+\operatorname{dim}\langle B\rangle-\operatorname{dim} L \geq 4,
$$

so $X \subset \mathbb{P}^{5}$ is the Veronese surface by Theorem 1.5 since $A=\mathbb{P}^{1}$ in the scroll case. If $n \geq 3$, we reduce to $n=2$ by cutting $X$ with $n-2$ general hyperplanes. To 
prove a) just note that the Veronese surface is not a hyperplane section of a smooth 3-fold.

Consider now $\operatorname{dim} L=n-1$. Then $\operatorname{dim}\langle B\rangle=N-2$, so there is a pencil $h_{\lambda} \subset \mathbb{P}^{N *}$ of hyperplanes containing $\langle B\rangle$. We can assume that $L$ is not a fixed component of $|V-B|$ (otherwise we argue with $|V-B-L|)$.

Claim 1. A general $R_{\lambda} \in|V-B|$ is smooth in codimension one.

By Theorem 1.1, a general $R_{\lambda}$ is smooth away from the base locus $\operatorname{Bs}|V-B|$ of $|V-B|$. So, if $\operatorname{Bs}|V-B|=\emptyset$, then a general $R_{\lambda}$ is smooth. Assume now that $\mathrm{Bs}|V-B| \neq \emptyset$ and take a point $x \in \mathrm{Bs}|V-B|$. Suppose by contradiction that $x$ is a singular point of a general $R_{\lambda} \in|V-B|$. Since this is a closed condition, we deduce that $x$ is a singular point of $R_{\lambda}$ for every $\lambda \in \mathbb{P}^{1}$. From Remark 1.2, it follows that $h_{\lambda}$ is a pencil of tangent hyperplanes at $x$. Therefore the embedded tangent $n$-plane $\mathbb{T}_{x} X$ to $X$ at $x$ is contained in the base locus $\langle B\rangle$ of $h_{\lambda}$. In particular $x$ is a singular point of $A=R_{\lambda_{0}}$, so

$$
\langle A\rangle=\mathbb{T}_{x} X \subset\langle B\rangle
$$

contradicting the hypothesis about $L$.

Claim 2. $R_{\lambda}$ is a hypersurface for every $\lambda \in \mathbb{P}^{1}$.

Let $K \subset \mathbb{P}^{N}$ be a general linear subspace of codimension $n-2$. For a general $\lambda \in \mathbb{P}^{1}$ we have that $R_{\lambda} \cap K$ is a smooth curve by claim 1 , so

$$
g\left(R_{\lambda} \cap K\right)=p_{a}\left(R_{\lambda} \cap K\right)=p_{a}(A \cap K),
$$

where $g$ and $p_{a}$ denote respectively the geometric and arithmetic genus. The second equality follows from $A \in\left|R_{\lambda}\right|$. Since $A \cap K$ is a plane curve, then $R_{\lambda} \cap K$ is also a plane curve in view of the Castelnuovo bound. Therefore a general (and hence every) $R_{\lambda}$ is also a hypersurface.

Thus $X \subset \mathbb{P}^{N}$ contains a pencil of hypersurfaces, so $X$ is a divisor on a rational scroll of $n$-planes and b) is proved.

For $\operatorname{dim} L=n$, as in Corollary 2.5. we can only determine $X$ if $B$ is also a hypersurface.

Theorem 3.4. Let $X \subset \mathbb{P}(V)=\mathbb{P}^{N}$ be an $n$-fold having a reducible divisor $A+B \in$ $|V|$. If $\langle A\rangle=\mathbb{P}^{n}$ and $\langle B\rangle=\mathbb{P}^{n}$, then one of the following holds:

i) $X \subset \mathbb{P}^{5}$ is the Veronese surface or its general projection to $\mathbb{P}^{4}$;

ii) $n=2$ and $X \subset \mathbb{P}^{4}$ is either a c.i. or linked to a 2-plane by a c.i. of a quadric cone of rank 4 and another hypersurface of $\mathbb{P}^{4}$;

iii) $n=2$ and $X \subset \mathbb{P}^{4}$ is linked to a 2-plane by a c.i. of a quadric cone of rank 3 and another hypersurface of $\mathbb{P}^{4}$;

iv) $n=3$ and $X \subset \mathbb{P}^{5}$ is linked to a 3-plane by a c.i. of a quadric cone of rank 4 and another hypersurface of $\mathbb{P}^{5}$;

v) $n=2, X \subset \mathbb{P}^{3}$ and $\operatorname{deg} X \geq 4$.

Proof. By Lemma 2.1 it follows that $n-2 \leq \operatorname{dim} L \leq n$. If $n-2 \leq \operatorname{dim} L \leq n-1$, then, by Proposition 3.3, either $X \subset \mathbb{P}^{5}$ is the Veronese surface or $X \subset \mathbb{P}^{n+2}$ is a divisor on a rational scroll of $n$-planes. Except for the projected Veronese surface in $\mathbb{P}^{4}$, an $n$-fold $X \subset \mathbb{P}^{n+2}$ is linearly normal by Zak's theorem (9], II Cor. 2.17), so either case i) holds or $X$ is a divisor on a rational normal scroll of $n$-planes of degree 2 (cf. Example 2.2). 
So we suppose $X \subset \mathbb{P}^{n+2}$ to be a smooth divisor on a quadric cone $Q$ of rank 3 or 4 . Therefore $X$ is either a c.i. or linked to an $n$-plane by a c.i. (see [4, Th. 2.1). Note that from Remark 1.8 it follows that $n \leq 3$. If $n=2$ and $X \subset \mathbb{P}^{4}$ is a c.i., the vertex $W$ of the cone cannot intersect $X$. Otherwise $X$ is singular along $X \cap W$. This fact together with the above considerations gives the cases ii) and iii). If $n=3$, then $X$ cannot be a c.i. by Remark 1.8 and, to get iv), we have to exclude the rank 3 case. By contradiction, suppose $X \subset \mathbb{P}^{5}$ to be contained in a quadric cone $Q$ of rank 3 , that is, $X \subset \mathbb{P}(\mathcal{E})$ for $\mathcal{E} \cong \mathcal{O}_{\mathbb{P}^{1}}^{\oplus 3} \oplus \mathcal{O}_{\mathbb{P}^{1}}(2)$. The vertex $W=\mathbb{P}^{2}$ of $Q$ is contained in $X$ by 4 , Cor. 2.7. Moreover, $W$ corresponds to $\tilde{W}:=\mathbb{P}\left(\mathcal{O}_{\mathbb{P}}^{\oplus 3}\right) \subset \mathbb{P}(\mathcal{E})$ and $X \cap \tilde{W}$ is isomorphic to $W=\mathbb{P}^{2}$. This implies that $X \cap \tilde{W}$ is a fiber of $\mathbb{P}\left(\mathcal{O}_{\mathbb{P}^{1}}^{\oplus 3}\right) \cong \mathbb{P}^{1} \times \mathbb{P}^{2}$, which is impossible since $X$ must intersect any fiber $\mathbb{P}^{2}$ of $\tilde{W}$ for dimensional reasons. Finally, if $\operatorname{dim} L=n$, then $X \subset \mathbb{P}^{n+1}$ is a hypersurface. So we get $n=2$ by Remark 1.8 and necessarily $\operatorname{deg} X \geq 4$.

Since $X \cong \mathbb{P}\left(\mathcal{O}_{\mathbb{P}^{1}}(1) \oplus \mathcal{O}_{\mathbb{P}^{1}}(2)\right) \subset \mathbb{P}^{4}$ (resp. $\left.X \cong \mathbb{P}\left(\mathcal{O}_{\mathbb{P}^{1}}(1)^{\oplus 3}\right) \subset \mathbb{P}^{5}\right)$ is linked to a 2-plane (resp. 3-plane) by the c.i. of two quadric hypersurfaces, we can summarize our work in the following way.

Corollary 3.5. Let $X \subset \mathbb{P}(V)=\mathbb{P}^{N}$ be an $n$-fold having a reducible divisor $A+B \in$ $|V|$. If $A \subset \mathbb{P}^{n}$ and $B \subset \mathbb{P}^{n}$, then one of the following holds:

i) $X \subset \mathbb{P}^{5}$ is the Veronese surface or its general projection to $\mathbb{P}^{4}$;

ii) $n=2$ and $X \subset \mathbb{P}^{4}$ is either a c.i. or linked to a 2-plane by a c.i. of a quadric cone of rank 4 and another hypersurface of $\mathbb{P}^{4}$;

iii) $n=2$ and $X \subset \mathbb{P}^{4}$ is linked to a 2-plane by a c.i. of a quadric cone of rank 3 and another hypersurface of $\mathbb{P}^{4}$;

iv) $n=3$ and $X \subset \mathbb{P}^{5}$ is linked to a 3-plane by a c.i. of a quadric cone of rank 4 and another hypersurface of $\mathbb{P}^{5}$;

v) $n=2$ and $X \subset \mathbb{P}^{3}$.

\section{REFERENCES}

1. W. Barth, Transplanting cohomology classes in complex projective space, Amer. J. Math. 92 (1970), 951-967. MR0287032(44:4239)

2. M.C. Beltrametti, K.A. Chandler, A.J. Sommese, Reducible hyperplane sections II, Kodai Math. J. 25 (2002), no. 2, 139-150. MR.1913521 (2003h:14012)

3. K.A. Chandler, A. Howard, A.J. Sommese, Reducible hyperplane sections I, J. Math. Soc. Japan 51 (1999), 887-910. MR.1705253 (2000g:14052)

4. S. Kwak, Castelnuovo-Mumford regularity bound for smooth threefolds in $\mathbb{P}^{5}$ and extremal examples, J. Reine Angew. Math. 509 (1999), 21-34. MR1679165 (2000e:14064)

5. A. Lanteri, A.L. Tironi, On reducible hyperplane sections of 4-folds, J. Math. Soc. Japan 53 (2001), no. 3, 559-563. MR1828969 (2002e:14009)

6. A. Lanteri, A.L. Tironi, Reducible hyperplane sections of threefolds: two components of sectional genus zero, Kodai Math. J. 27 (2004), no. 3, 299-320. MR2100925

7. M.E. Larsen, On the topology of projective manifolds, Invent. Math. 19 (1973), 251-260. MR0318511 (47:7058)

8. A. Van de Ven, On the 2-connectedness of very ample divisors on a surface, Duke Math. J. 46 (1979), no. 2, 403-407. MR0534058 (82f:14032)

9. F.L. Zak, Tangents and Secants of Algebraic Varieties, Translations of Mathematical Monographs 127, American Mathematical Society, Providence, RI, 1993. MR.1234494(94i:14053) 
Departamento de Álgebra, Facultad de Ciencias Matemáticas, Universidad ComPlutense De MAdRid, 28040 Madrid, Spain

E-mail address: jcsierra@mat.ucm.es

Dipartimento di Matematica "F. Enriques", Università degli Studi di Milano, Via C. Saldini 50, 20133 Milano, Italy

E-mail address: atironi@mat.unimi.it 